\title{
Programação competitiva como ferramenta de apoio ao ensino de algoritmos e estrutura de dados para alunos de Ciência da Computação
}

\author{
Palloma S. S. Brito ${ }^{1}$, Reinaldo S. Fortes ${ }^{1}$, Felipe L. de M. Faria ${ }^{2}$, \\ Rodolfo A. Lopes ${ }^{1}$, Valéria de C. Santos ${ }^{1}$, Felipe F. de Á. Magalhães ${ }^{1}$ \\ Departamento de Computação - Universidade Federal de Ouro Preto (UFOP) \\ Campus Universitário Morro do Cruzeiro - CEP 35400-000 - Ouro Preto - MG - Brasil \\ Departamento de Engenharia e Computação - Instituto Federal de Minas Gerais (IFMG) \\ Campus Bambuí - CEP 38.900-000 - Bambuí - MG - Brasil \\ \{palloma.brito, felipe.magalhaes\}@aluno.ufop.edu.br,rodolfo.ufop@gmail.com \\ \{reifortes, valeriacs\}@ufop.edu.br,felipe.fariadifmg.edu.br
}

\begin{abstract}
This article presents a methodology for teaching algorithms and data structure in Computer Science course based on the use of competitive programming as a tool to support the learning process, developing logical reasoning and stimulating teamwork. A comparative analysis between experimental and control groups reveals that students who used the proposed methodology had higher academic achievement in the course.
\end{abstract}

Resumo. Este artigo apresenta uma metodologia de aprendizagem para disciplinas de algoritmos e estrutura de dados no curso de Ciência da Computação baseada no uso da programação competitiva como ferramenta de apoio ao processo de ensino-aprendizagem, desenvolvendo o raciocínio lógico e estimulando o trabalho em equipe. Uma análise comparativa entre grupos experimental e de controle revela que os alunos que utilizaram a metodologia proposta possuíram rendimento acadêmico superior no curso.

\section{Introdução}

Maratonas de programação são competições destinadas a alunos da área de Computação e afins (Ciência da Computação, Engenharia de Computação, Sistemas de Informação, Matemática, etc). Tais competições estimulam a criatividade e o trabalho em equipe, além de desenvolverem o raciocínio lógico e a habilidade de resolver problemas sob pressão. Grandes empresas como Google e Facebook apresentam em suas entrevistas técnicas, problemas similares aos aplicados em maratonas de programação. Por isso é muito comum maratonistas e ex-maratonistas serem contratados com mais frequência.

Em Ciência da Computação, disciplinas relacionadas a algoritmos e estrutura de dados são lecionadas logo nos primeiros períodos do curso. Tais disciplinas são consideradas como as mais desafiadoras, visto que exigem o desenvolvimento de estratégias de solução de problemas com base lógico-matemática, atribuindo aos professores uma forte demanda de interação com os alunos, pois faz-se necessário identificar e auxiliar as dificuldades de cada um. Muitas vezes essas dificuldades não são detectadas e sanadas em tempo hábil, por motivos didático-organizacionais. 
De acordo com Gonçalves et al. (2013), o alto nível de abstração exigido em disciplinas relacionadas à programação causa desmotivação nos alunos, fator preponderante para altas taxas de reprovações e desistências. Diante do exposto, pode-se estimar que o uso de programação competitiva como parte do ensino de disciplinas de algoritmos e estrutura de dados afeta de maneira positiva o desenvolvimento acadêmico dos alunos. A ideia é utilizar competições de programação como motivação ao estudo de algoritmos e, assim, reduzir o número de reprovações e evasões.

O objetivo deste trabalho é apresentar uma metodologia de ensino de algoritmos e estrutura de dados, aplicada através de um encontro semanal e atividades extraclasse, aos alunos do curso de Ciência da Computação da Universidade Federal de Ouro Preto. A metodologia é baseada no uso da programação competitiva no processo de ensinoaprendizagem complementada por gamificação. Análise de resultados revela que os alunos que participaram das atividades apresentaram desempenho superior aos demais.

Este artigo está organizado como se segue. A Seção 2 apresenta os principais trabalhos relacionados a esta pesquisa. A Seção 3 apresenta os métodos que influenciaram o desenvolvimento deste trabalho. A Seção 4 aborda a metodologia de ensino utilizada. Na Seção 5 os resultados são discutidos. Por fim, a Seção 6 apresenta a conclusão.

\section{Trabalhos Relacionados}

Além da inclusão da lógica de programação na educação básica, existem diversas outras estratégias que buscam motivar o processo de aprendizagem em disciplinas de algoritmos e estrutura de dados. O Scratch é uma das ferramentas mais utilizadas na introdução à lógica de programação nos últimos anos (Borges et al. , 2018). Desenvolvida pelo Lifelong Kindergarten Group do Laboratório de Mídias do Massachusetts Institute of Technology (MIT), visa desenvolver habilidades de programação de maneira lúdica e criativa, sendo utilizada majoritariamente em crianças do ensino básico. O Block-C é uma ferramenta baseada no Scratch que permite que a programação seja feita através da manipulação direta de blocos visuais, além de oferecer suporte à rígida linguagem $\mathrm{C}$. $\mathrm{O}$ trabalho realizado por Kyfonidis et al. (2017) revela alto nível de satisfação dos alunos com o uso do Block-C, entretanto não houveram distinções, em termos de rendimento acadêmico, entre usuários e não usuários da ferramenta. Uma experimentação mais intensiva é necessária para investigar os efeitos a longo prazo do uso dessa ferramenta.

A técnica de PBL (Problem Based Learning) é um método de ensino centrado no aluno, consistindo, basicamente, na resolução de exercícios como forma de aprendizagem. Nela, os professores passam de uma posição que fornece o conhecimento para uma posição orientadora, estimulando o raciocínio lógico e a autonomia. Em disciplinas de algoritmos e estrutura de dados, essa técnica pode ser associada ao URI Online Judge, ferramenta online que permite praticar a programação e compartilhar o conhecimento.

O trabalho desenvolvido por Ambrósio \& Costa (2010) utiliza a técnica de PBL como parte da metodologia de ensino para a disciplina de Algoritmos e Programação de Computadores. Como adicional, a metodologia faz uso de tablets e do trabalho colaborativo. Antes de implementar um algoritmo em uma linguagem de programação, os alunos devem se concentrar na estruturação do algoritmo que fornecerá a solução para o problema. Além do aspecto motivacional, os tablets representam uma importante ferramenta de colaboração, servindo como base de pesquisa e como um meio de anotações. 
VIII Congresso Brasileiro de Informática na Educação (CBIE 2019)

Anais do XXX Simpósio Brasileiro de Informática na Educação (SBIE 2019)

Uma das apostas do século XXI na área educacional envolve o uso da gamificação para engajar os alunos a atingirem um determinado objetivo. Sgoti \& Mill (2018) exemplificam o uso da gamificação através da criação de uma lista de exercícios para a disciplina de algoritmos utilizando sistemas de recompensa e bonificação. Além de motivar os alunos, a lista gamificada alcançou um maior índice de aproveitamento na resolução dos exercícios quando comparada à lista de exercícios convencional. Outros trabalhos, como em Carreño-León et al. (2018) e Silva et al. (2016), também fizeram uso da gamificação como parte da metodologia de ensino e obtiveram resultados similares. Butler \& Ahmed (2016) propuseram o desenvolvimento de um jogo para introduzir conceitos apresentados na disciplina de estruturas de dados (pilhas, filas e ordenação por seleção). Apesar de os alunos afirmarem que essa metodologia de ensino torna a aprendizagem mais divertida e interativa, os jogos convencionais ainda são mais atrativos que os educativos.

Piekarski et al. (2015) apresentaram uma metodologia de ensino muito similar à proposta neste trabalho. Eles se baseiam no emprego de elementos presentes nas maratonas de programação (sistema de ranqueamento, resolução de problemas e competições simuladas) como forma de desenvolvimento do raciocínio lógico e do trabalho colaborativo. Além de desenvolver habilidades nas linguagens de programação, a introdução dessa metodologia estimulou os alunos a resolverem problemas aplicados nas competições, sendo esses os principais resultados alcançados.

Todos os autores citados anteriormente apresentam possíveis metodologias de ensino para disciplinas de algoritmos e estrutura de dados com o intuito de amenizar as elevadas taxas de reprovação, desistência e evasão em cursos de computação. Entretanto, não há uma avaliação sistemática sobre os efeitos causados após implantação de tais metodologias. Nesse trabalho, será avaliado o desenvolvimento acadêmico dos alunos que utilizaram a programação competitiva como ferramenta de apoio em disciplinas de programação.

\section{Materiais e métodos}

A metodologia deste trabalho é definida com base nos conceitos de maratona de programação, gamificação e da utilização de uma plataforma online que permita acesso a problemas, submissão de soluções e correção automática das soluções submetidas. Nas próximas subseções são apresentados cada um destes conceitos e recursos.

\subsection{Maratonas de Programação}

As maratonas de programação são competições destinadas a alunos da área de Computação e afins. As competições são realizadas em grupos de três pessoas e possui cerca de 11 problemas que podem ser resolvidos em no máximo 5 horas. Cada problema possui um balão com uma cor. Quando uma solução é aceita, isto é, apresenta a resposta correta para todas as instâncias do problema, a equipe recebe um balão com a cor relativa ao problema.

A equipe que realiza o maior número de problemas em menos tempo vence. Em caso de empates quanto ao número de problemas resolvidos, vence a equipe que possui menor penalidade. Inicialmente, todas as equipes possuem penalidade igual a zero. Quando uma equipe resolve um problema no tempo $T$, sua penalidade é acrescida de $T$ pontos. Além disso, para cada submissão errada de um determinado problema, a penalidade é acrescida de 20 pontos, que são computados assim que a solução é aceita. 
VIII Congresso Brasileiro de Informática na Educação (CBIE 2019)

Anais do XXX Simpósio Brasileiro de Informática na Educação (SBIE 2019)

A participação nas maratonas deve ser realizada sem nenhum acesso à internet e/ou dispositivos eletrônicos, entretanto é permitido consultar qualquer tipo de material didático impresso.

\subsection{Gamificação}

Segundo Deterding et al. (2011), gamificação se refere ao uso de design, elementos, características de jogos em contextos que não são de jogos. Fiqueiredo et al. (2015) afirma que a gamificação refere-se ao uso de mecanismos e dinâmicas de jogos para a resolução de problemas e para a motivação e o engajamento de um público em uma atividade ou tarefa. A gamificação encontra na educação formal uma área fértil para sua aplicação, pois nela encontram-se indivíduos que aprendem com as interações com os jogos, além de ser uma área carente de estratégias para lidar com indivíduos que estão cada vez mais inseridos no contexto das tecnologias digitais e se mostram desinteressados pelos métodos passivos de ensino e aprendizagem utilizados na maioria das escolas (Fardo, 2013).

Ainda segundo Fardo (2013), características como distribuir pontuações para atividades, apresentar feedback e encorajar a colaboração em projetos são as metas de muitos planos pedagógicos. A diferença é que a gamificação possibilita uma camada mais explícita de interesse e um método para integrar esses elementos objetivando a similaridade com os games, resultando em uma linguagem com a qual os indivíduos inseridos na cultura digital estão mais acostumados. Como resultado, tende-se a conseguir alcançar essas metas de forma aparentemente mais eficiente e agradável

O Ministério da Educação, por exemplo, de acordo com Alves (2014) apoia o desenvolvimento de ambientes gamificados, como o Geekgames, uma plataforma online de aprendizado adaptativo que possibilita aos estudantes prepararem-se para o Exame Nacional do Ensino Médio (ENEM), por meio de desafios. Os inscritos têm acesso a um diagnóstico e a um estudo personalizado que possibilitam identificar suas limitações e acompanhar os avanços nas áreas a serem avaliadas pelo ENEM.

\subsection{Portal URI Online Judge}

O URI Online Judge é uma plataforma online e gratuita que permite praticar a programação de maneira competitiva e gamificada. A plataforma possui uma vasta quantidade de exercícios que são agrupados de acordo com o assunto abordado nos mesmos. Dentre suas inúmeras funcionalidades, destacam-se:

- correção automática em tempo real das soluções submetidas;

- discussão dos problemas através de fóruns, possibilitando o compartilhamento de conhecimentos;

- aceitação de soluções em diversas linguagens de programação (como C, C++ e Python);

- depuração integrada para verificar saídas para instâncias de entradas adicionais;

- separação dos problemas em categorias e níveis de dificuldades;

- aplicação de conceitos de gamificação, como rankings e sistema de recompensas;

- visualização dos erros encontrados na compilação e execução de uma solução;

- criação de competiçõos simuladas;

- repositório das soluções submetidas. 
VIII Congresso Brasileiro de Informática na Educação (CBIE 2019)

Anais do XXX Simpósio Brasileiro de Informática na Educação (SBIE 2019)

\section{Metodologia de ensino}

A metodologia de ensino aplicada é baseada em um fluxograma de atividades conforme apresentado na Figura 1. As etapas são descritas nas subseções a seguir.

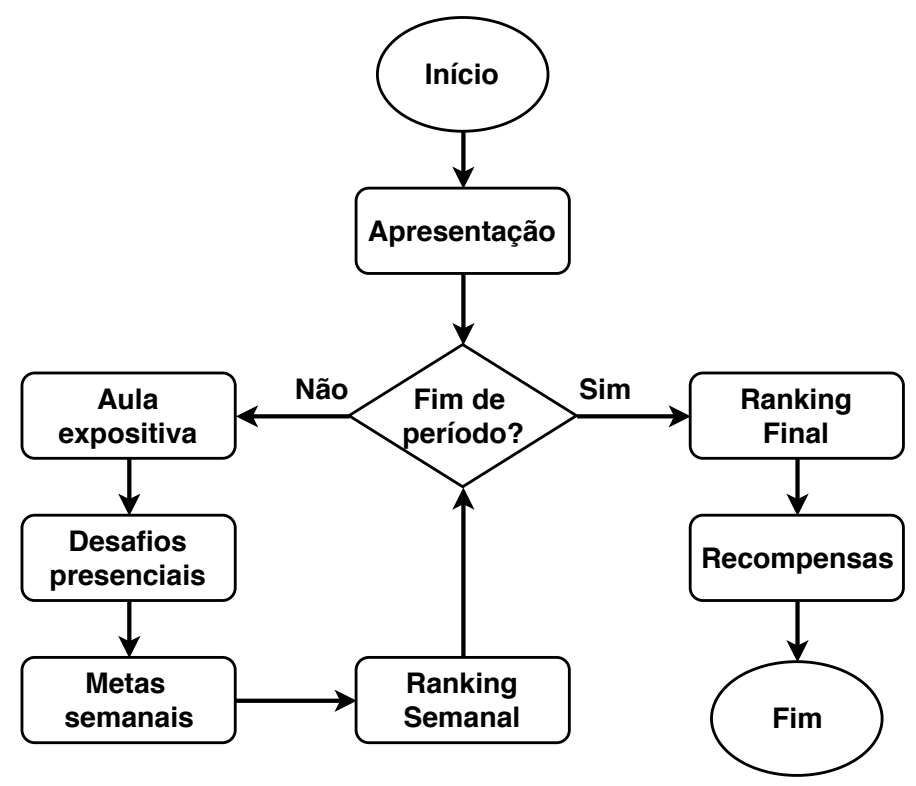

Figura 1. Fluxograma da metodologia de ensino.

\subsection{Apresentação}

A metodologia é baseada, em primeiro lugar, na adesão voluntária dos alunos dos primeiros períodos do curso de Ciência da Computação que ainda estejam cursando uma das disciplinas de algoritmos ou estruturas de dados. O programa não é integrado diretamente às disciplinas, ou seja, não faz parte do plano de ensino das mesmas.

Sendo assim, com o apoio dos professores das disciplinas relacionadas, é feita uma apresentação aos alunos, explicando a importância da prática constante de programação para o aprendizado, a importância da programação para o curso de Ciência da Computação, como as atividades são conduzidas e mais detalhes sobre o programa. Em seguida, os alunos interessados se inscrevem através de formulário online.

\subsection{Aulas Expositivas e Desafios Presenciais}

Os encontros presenciais são realizados durante o período letivo. Cada encontro é dividido em: (1) uma aula expositiva, que reforça o conteúdo abordado em sala de aula e introduz novos conceitos de programação e algoritmos; e (2) desafios presenciais, que consiste da resolução de problemas de programação.

Os temas apresentados vão desde dicas sobre as estruturas aceitas nas maratonas de programação até o aprofundamento nas linguagens de programação e problemas de lógica utilizadas nesses eventos.

Os encontros presenciais são realizados semanalmente em um dos laboratórios de informática da universidade, onde se utiliza o Portal do URI como ferramenta de apoio à prática da programação. Através dessa plataforma são realizados torneios que simulam as competições de programação. Os alunos são desafiados a trabalharem em equipe 
para resolverem problemas específicos, seguindo as mesmas regras das maratonas de programação. As equipes vencedoras são premiadas com medalhas e outras bonificações.

Os elementos da gamificação presentes do Portal URI despertam o espírito competitivo dos alunos fazendo com que eles pratiquem a programação fora do ambiente acadêmico. Eles se sentem desafiados a resolverem mais problemas para, assim, ocuparem as primeiras colocações no ranking da universidade.

\subsection{Metas e Ranking Semanais}

Além dos encontros presenciais, a cada semana os alunos são instigados a cumprirem determinadas metas, que consistem basicamente de um conjunto de problemas disponibilizados no Portal URI. Exercícios relacionados aos tópicos abordados nas disciplinas e nos encontros presenciais são selecionados com o cuidado de reforçar o aprendizado e manter a prática constante da programação.

Paralelo ao ranking individual do URI, são formadas equipes de três alunos e é computado e disponibilizado um ranking semanal, atualizado de acordo com a frequência ao encontro presencial e ao desempenho nos desafios e metas semanais. Estes rankings têm como intuito estimular os alunos a manter as atividades através de uma competitividade saudável entre os participantes.

\subsection{Ranking Final e Recompensas}

No final do período, após o encerramento dos encontros semanais, é computado um ranking final das equipes participantes. O engajamento ao longo do período letivo é estimulado através do espírito competitivo dos alunos com o objetivo de atingirem uma boa colocação no ranking final e, com isso, desfrutarem de algumas recompensas:

- A equipe vencedora normalmente é agraciada com um jantar oferecido por um restaurante parceiro.

- Os alunos recebem pontuação extra nas disciplinas, em forma de trabalho prático, ponderado pelas atividades desenvolvidas e a posição no ranking.

- Os alunos recebem certificados que lhes garantem aproveitamento em horas de atividade extraclasse, obrigatórias para a integralização de créditos.

\section{Resultados}

Visando avaliar a metodologia de ensino proposta neste trabalho, os dados de 73 alunos divididos em dois grupos (experimental e controle) foram avaliados. Dentre estes alunos, $60 \%$ aderiram ao programa proposto, representando o grupo experimental, e $40 \%$ não aderiram, representando o grupo de controle. Foram considerados apenas alunos matriculados nas turmas em períodos nos quais o programa foi executado.

Nesta seção é apresentada uma análise do desempenho dos alunos nas disciplinas de algoritmos e estruturas de dados, na Seção 5.1, e uma análise geral do histórico de disciplinas cursadas pelos alunos, na Seção 5.2. O intuito dessa divisão é buscar compreender se há informações que corroboram para a ideia de que a metodologia proposta neste estudo pode colaborar no desempenho acadêmico dos alunos do curso de Ciência da Computação de maneira geral, e não apenas nas disciplinas de algoritmos e estrutura de dados. Na Seção 5.3 são apresentados os resultados de uma pesquisa de opinião aplicada aos alunos que participaram do programa. 
VIII Congresso Brasileiro de Informática na Educação (CBIE 2019)

Anais do XXX Simpósio Brasileiro de Informática na Educação (SBIE 2019)

\subsection{Disciplinas de algoritmos e estruturas de dados}

Avaliou-se o desempenho dos alunos nas disciplinas de algoritmos e estruturas de dados. A Tabela 1 descreve o desempenho dos alunos separando-os em aprovados e reprovados. São classificados como aprovados aqueles alunos cuja média final da disciplina é maior ou igual a 6,0 . alunos classificados com reprovados são aqueles cuja média final é inferior a 6,0 e/ou sua frequência é menor que $75 \%$ das aulas ministradas.

\begin{tabular}{|c|c|c|}
\hline & Alunos & Nota Média \\
\hline Aprovados & $49(67,12 \%)$ & 7,97 \\
\hline Reprovados & $24(32,87 \%)$ & 2,31 \\
\hline
\end{tabular}

\section{Tabela 1. Desempenho geral dos alunos nas disciplinas de algoritmos e estrutu- ras de dados.}

No contexto dos grupos experimental e de controle, uma relação do desempenho final nas disciplinas de algoritmos e estruturas de dados é descrita na Tabela 2. Identificase uma diferença significativa de performance quando comparando alunos do grupo experimental e de controle. O percentual de alunos aprovados no grupo experimental é superior ao grupo de controle em 25,55 pontos percentuais e a nota média é superior em 1,68 pontos. Em relação aos reprovados, por sua vez, além do percentual inferior, a nota média dos alunos do grupo experimental é superior aos alunos do grupo de controle em 2,31 pontos.

\begin{tabular}{|c|c|c|c|c|}
\hline \multirow{2}{*}{} & \multicolumn{2}{|c|}{ Experimental } & \multicolumn{2}{c|}{ Controle } \\
\cline { 2 - 5 } & Alunos & Nota Média & Alunos & Nota Média \\
\hline Aprovados & $34(77,27 \%)$ & 8,19 & $15(51,72 \%)$ & 7,51 \\
\hline Reprovados & $10(22,72 \%)$ & 3,66 & $14(48,27 \%)$ & 1,35 \\
\hline
\end{tabular}

\section{Tabela 2. Desempenho dos alunos dos grupos experimental e de controle nas disciplinas de algoritmos e estruturas de dados}

Correlacionando os dados das Tabelas 1 e 2 é possível observar que os alunos do grupo experimental contribuíram significativamente para a melhoria do desempenho geral dos alunos em relação às disciplinas de algoritmos e estruturas de dados, não somente em relação à quantidade de alunos, mas também em relação às notas médias. Note que a diferença entre a nota média dos alunos aprovados do grupo experimental e a nota média geral é de 0,22 pontos, enquanto a diferença entre a nota média do grupo de controle e a nota média geral é de 0,76 . Por outro lado, as diferenças entre as notas médias dos alunos reprovados do grupo experimental e do grupo de controle em relação à nota média geral são de 1,35 e 0,96 , respectivamente.

\subsection{Histórico Completo}

Avaliou-se também o desempenho dos alunos em relação a todo o histórico de disciplinas cursadas. A Tabela 3 apresenta informações sobre os alunos aprovados e reprovados 
VIII Congresso Brasileiro de Informática na Educação (CBIE 2019)

Anais do XXX Simpósio Brasileiro de Informática na Educação (SBIE 2019)

\begin{tabular}{|c|c|c|}
\hline & Porcentagem & Média das Notas \\
\hline Aprovados & $72,54 \%$ & 7,86 \\
\hline Reprovados & $27,45 \%$ & 1,81 \\
\hline
\end{tabular}

Tabela 3. Desempenho geral dos alunos em todas as disciplinas cursadas.

dentre todas as disciplinas por eles cursadas, seguindo os mesmos critérios de aprovação utilizados na Seção 5.1 .

No contexto dos grupos experimental e de controle, uma relação do desempenho final em todas as disciplinas cursadas é descrita na Tabela 4. Embora o grupo experimental claramente apresente resultados melhores, identifica-se uma diferença de performance mais discreta em relação às disciplinas mais específicas avaliadas anteriormente, quando comparado aos alunos do grupo de controle. O percentual de alunos aprovados no grupo experimental é superior ao grupo de controle em 17,34 pontos percentuais e a nota média é superior em 0,16 pontos. Em relação aos reprovados, por sua vez, além do percentual inferior, a nota média dos alunos do grupo experimental é superior aos alunos do grupo de controle em 0,71 pontos.

\begin{tabular}{|c|c|c|c|c|}
\hline \multirow{2}{*}{} & \multicolumn{2}{|c|}{ Experimental } & \multicolumn{2}{c|}{ Controle } \\
\cline { 2 - 5 } & Alunos & Nota Média & Alunos & Nota Média \\
\hline Aprovados & $79,59 \%$ & 7,92 & $62,25 \%$ & 7,76 \\
\hline Reprovados & $20,40 \%$ & 2,21 & $37,74 \%$ & 1,50 \\
\hline
\end{tabular}

\section{Tabela 4. Desempenho dos alunos dos grupos experimental e de controle em todas as disciplinas cursadas.}

Correlacionando os dados das Tabelas 3 e 4 é possível observar que a influência da participação no programa foi menos significativa quando considerando todas as disciplinas cursadas. Note que a diferença entre a nota média dos alunos aprovados do grupo experimental e a nota média geral é de 0,06 pontos, enquanto a diferença entre a nota média do grupo de controle e a nota média geral é de 0,10 . Por outro lado, as diferenças entre as notas médias dos alunos reprovados do grupo experimental e do grupo de controle em relação à nota média geral são de 0,40 e 0,31 , respectivamente.

Este resultado pode ser consequência do programa ter sido iniciado recentemente, no segundo semestre de 2017, sendo assim, os alunos ainda se encontram na primeira metade do curso, momento em que ainda não estão cursando disciplinas básicas e não cursaram a maioria das disciplinas técnicas da área de Ciência da Computação.

\subsection{Questionário de opinião}

Nas subseções anteriores apresentou-se evidências numéricas de que a participação no programa pode influenciar positivamente o desempenho dos alunos em suas atividades acadêmicas. Entretanto, vários fatores não controlados podem influenciar os resultados, impossibilitando comprovar causalidade. 
VIII Congresso Brasileiro de Informática na Educação (CBIE 2019)

Anais do XXX Simpósio Brasileiro de Informática na Educação (SBIE 2019)

Sendo assim, realizou-se uma pesquisa de opinião junto aos alunos do grupo experimental, ou seja, aqueles que participaram do programa. A Tabela 5 apresenta o percentual de respostas obtido para cada pergunta, as respostas permitidas eram valores inteiros entre 1 e 5 , quanto maior o valor, maior a satisfação do aluno.

\begin{tabular}{|l|c|c|c|c|c|}
\hline Perguntas \Respostas & 1 & 2 & 3 & 4 & 5 \\
\hline Satisfação com a apresentação inicial & $0 \%$ & $0 \%$ & $20 \%$ & $40 \%$ & $40 \%$ \\
\hline Satisfação com o conteúdo abordado & $0 \%$ & $0 \%$ & $10 \%$ & $20 \%$ & $70 \%$ \\
\hline Relevância para o aprendizado no curso & $0 \%$ & $10 \%$ & $30 \%$ & $20 \%$ & $40 \%$ \\
\hline
\end{tabular}

Tabela 5. Questionário de opinião aplicado aos alunos do grupo experimental.

Observa-se um grande percentual de alunos que se sentiram satisfeitos com a apresentação inicial do programa, algo que explica a adesão de mais da metade dos alunos matriculados nas disciplinas de algoritmos e estruturas de dados. Também é possível observar que a maioria dos alunos também considera que o conteúdo foi abordado de maneira adequada e que a sua participação no programa foi relevante para o aprendizado no curso de Ciência da Computação. Quanto à relevância para o aprendizado, observa-se $10 \%$ apresentaram resposta 2 e $30 \%$ para a resposta 3 , que pode ser considerado um ponto a ser considerado em trabalhos futuros visando obter melhoria nestes indicadores.

Somando os resultados numéricos às respostas apresentadas pelos alunos do grupo experimental pode-se concluir que de fato o programa contribuiu para a melhoria de desempenho acadêmico dos alunos participantes.

\section{Conclusão}

Neste trabalho, foi apresentada uma metodologia de ensino que tem como componente central a programação competitiva como ferramenta de apoio pedagógico para a melhoria no desempenho acadêmico dos alunos no curso de Ciência da Computação de uma universidade federal.

Análises comparativas entre os alunos que participaram do programa e aqueles que não participaram revelam que a metodologia apresentada de fato contribuiu para um melhor desempenho acadêmico dos alunos nas disciplinas de algoritmos e estruturas de dados e possui potencial para melhorar seu desempenho geral no curso.

Dificuldades no controle de fatores externos que podem influenciar no desempenho dos alunos dificultam comprovar causalidade e, levando em consideração que o programa foi iniciado recentemente, observa-se a necessidade de continuar as atividades junto aos novos alunos e continuar avaliando o desempenho dos alunos participantes ao longo de sua trajetória acadêmica. Como trabalhos futuros, objetiva-se propor novas métricas para avaliação dos grupos de estudo, aumentar o número de amostras para análise e aprimorar o questionário de opinião a fim de obter resultados mais abrangentes e conclusivos, além de propor adaptações que se fizerem necessárias à metodologia.

\section{Agradecimentos}

O presente trabalho foi realizado com apoio da Pró-Reitoria de Extensão (PROEX) da Universidade Federal de Ouro Preto (UFOP), projeto PRJ1359. 
VIII Congresso Brasileiro de Informática na Educação (CBIE 2019)

Anais do XXX Simpósio Brasileiro de Informática na Educação (SBIE 2019)

\section{Referências}

Alves, Lynn Rosalina Gama; Minho, Marcelle Rose da Silva; Diniz Marcelo Vera Cruz. 2014. Gamificação: diálogos com a educação. Chap. 3, pages 74-97 of: FADEL, Luciane Maria et al.(Org.) (ed), Gamificação na educação. São Paulo: Pimenta Cultural.

Ambrósio, Ana Paula L, \& Costa, Fábio M. 2010. Evaluating the impact of pbl and tablet pcs in an algorithms and computer programming course. Pages 495-499 of: Proceedings of the 41st ACM technical symposium on Computer science education. ACM.

Borges, R. P., Oliveira, P. R. F., Lima, R. G. R., \& Lima, R. W. 2018. A Systematic Review of Literature on Methodologies, Practices, and Tools for Programming Teaching. IEEE Latin America Transactions, 16(5), 1468-1475.

Butler, S., \& Ahmed, D. T. 2016 (Dec). Gamification to Engage and Motivate Students to Achieve Computer Science Learning Goals. Pages 237-240 of: 2016 International Conference on Computational Science and Computational Intelligence (CSCI).

Carreño-León, M., Sandoval-Bringas, A., Álvarez Rodríguez, F., \& Camacho-González, Y. 2018 (April). Gamification technique for teaching programming. Pages 2009-2014 of: 2018 IEEE Global Engineering Education Conference (EDUCON).

Deterding, Sebastian, Dixon, Dan, Khaled, Rilla, \& Nacke, Lennart. 2011. From Game Design Elements to Gamefulness: Defining "Gamification". Pages 9-15 of: Proceedings of the 15th International Academic MindTrek Conference: Envisioning Future Media Environments. MindTrek'11. New York, NY, USA: ACM.

Fardo, M. L. 2013. A gamificação aplicada em ambientes de aprendizagem: Novas Tecnologias na Educação. In: Cinted/UFRGS, vol. 11.

Fiqueiredo, Mercia, Paz, Tatiana, \& Junqueira, Eduardo. 2015 (10). Gamificação e educação: um estado da arte das pesquisas realizadas no Brasil.

Gonçalves, Dimas Antônio Silveira, da Silva, Gislaine Moura, da Luz, Ronaldo Santos, \& Silva, Eduardo Paulino. 2013. Relato de experiência de alunos do curso de Licenciatura em Computação do IFMG-campus Ouro Branco na utilização de objetos de aprendizagem desplugados e do Scratch como instrumentos no ensino de programação. In: Anais dos Workshops do Congresso Brasileiro de Informática na Educação, vol. 2.

Kyfonidis, C., Moumoutzis, N., \& Christodoulakis, S. 2017 (April). Block-C: A blockbased programming teaching tool to facilitate introductory $\mathrm{C}$ programming courses. Pages 570-579 of: 2017 IEEE Global Engineering Education Conference (EDUCON).

Piekarski, Ana Elisa, Miazaki, Mauro, Hild, Tony, Mulati, Mauro Henrique, \& Kikuti, Daniel. 2015. A metodologia das maratonas de programação em um projeto de extensão: um relato de experiência. Page 1246 of: Anais dos Workshops do Congresso Brasileiro de Informática na Educação, vol. 4.

Sgoti, Rogerio Ferreira, \& Mill, Daniel. 2018. GAMIFICAÇÃO DE UMA LISTA DE EXERCÍCIOS DA DISCIPLINA ALGORITMOS E ANÁLISE DE SUA APLICAÇÃO EM SUBSTITUIÇÃO AO MODO CONVENCIONAL. CIET: EnPED.

Silva, Tatyane, Melo, Jeane, \& Tedesco, Patrícia. 2016. Um modelo para promover o engajamento estudantil no aprendizado de programação utilizando gamification. Page 71 of: Anais dos Workshops do Congresso Brasileiro de Informática na Educação, vol. 5 . 